\title{
STUDIES ON PNEUMONIA IN CHILDREN
}

\section{Mortality, Blood Cultures, and Humoral Antibodies in Pneumococcus Pneumonia}

BY JAMES D. TRASK, CHARLES O'DONOVAN, JR., DOROTHEA M. MOORE AND AGNES R. BEEBE

(From the Department of Pediatrics, Yale University School of Medicine and the Pediatric Service of the New Haven Hospital and Dispensary, New Haven)

(Received for publication December 31, 1929)

Since the development $(1,2)$ of the biological classification of pneumococci bacteriological methods have been applied by many investigators (3-16) to the study of the incidence, mortality and course of lobar pneumonia. In uncomplicated cases certain phenomena have been found to be associated with recovery, others with death as shown in the following tabulation.

$\begin{array}{ll}\text { 1. Blood cultures........... Negative, or becoming } & \begin{array}{c}\text { Decoery } \\ \text { so }\end{array} \\ \begin{array}{c}\text { Becoming increasingly } \\ \text { positive }\end{array}\end{array}$

2. Specific soluble substances

in serum and urine..... Not detectable or present in small amounts

Increasing amounts in urine; occasionally detectable in serum

3. Type specific humoral antibodies.............. Detectable Not detectable

4. Excess of specific antibodies in serum of treated cases.......... Easily maintained

Difficult or impossible to maintain

The associations of these phenomena with the outcome of the disease have been so regular as to indicate the presence of a causal relation between them. Whatever the relation, it provides in large part the rationalization of type specific antipneumococcus serum therapy. The studies alluded to have been made largely on adults and the results have not been correlated with age.

It is stated in text books of medicine, and it appears to be the experience of practicing physicians that the case fatality rate of lobar pneu- 
monia is lower in children than in adults. In 2,000 typed cases of pneumococcus pneumonia, Cecil, Baldwin and Larsen (4) found, in general, that in adults the younger the patient the better the prognosis. Blake (17) observed in studying pneumococcus Type III pneumonia a considerable number of mild cases of short duration in young adults, whereas the severe conventional form of Type III pneumonia was seen chiefly in those over 40 years of age. Consequently, it would be interesting to learn whether the measurable factors which presumably influence the outcome in adults differ in children from those found in older persons. The present study was, therefore, undertaken and directed along familiar lines, as follows: (1) the collection of data on the mortality in pneumonia due to the fixed types of pneumococcus in children and in adults in the same locality and during the same years; (2) the study in children with pneumonia of $(a)$ the presence of pneumococci in the blood and $(b)$ the development of type specific humoral antibodies.

\section{MATERIAL AND METHODS}

Material. The material was collected from all cases diagnosed as pneumonia on all services of The New Haven Hospital from July, 1927, to June, 1929, inclusive. The cases were reviewed and included, if it was felt that an acute bronchogenetic infection of the lung parenchyma had been present. The differentiation of lobar and bronchopneumonia was made on clinical grounds and $\mathrm{x}$-ray evidence, uninfluenced, as far as possible, by bacteriological findings. Some cases of illdefined bronchopneumonia, terminating chronic illness and following operation, are included among the adults; only a few similar cases are found among the children. The bulk of the material is from the medical and pediatric services and consists of classical cases of lobar and bronchopneumonia. Those twelve years and younger were pediatric patients, and are called children, those older are classed as adults. Twenty autopsies were obtained in children, thirty-six in adults.

Methods. We are indebted to the Medical Service for the clinical notes and the etiological diagnosis of adult cases, determined in the usual ways,--by routine cultures and mouse inoculation of sputum, routine blood cultures, precipitin tests on urine and examination of exudates. The same procedures were employed in the pediatric laboratory, slightly modified for application to children. Since sputum was difficult to obtain, material obtained by swabbing the throat was substituted. The pharynx was thoroughly swabbed with a sterile applicator armed with cotton. This was quickly washed in $5 \mathrm{cc}$. of broth and $1 \mathrm{cc}$. of the washing was injected into a white mouse. The mice were autopsied in the usual way. If they survived seven days they were discarded. Some mice dying after five or six days yielded pure cultures of pneumococcus Type I. Rarely, when the 
diagnosis was made by a positive blood culture, the mice were not autopsied. For blood cultures, 5 to $10 \mathrm{cc}$. of blood was inoculated into 100 to $150 \mathrm{cc}$. of broth at the bedside. Blood was also added to an equal volume of citrate solution (1.5 per cent) and 1 and $2 \mathrm{cc}$. pour plates were then made in the laboratory. The remainder of the blood was allowed to clot, the serum recovered and saved in "no-air" rubber stoppered bottles in the refrigerator. The clot was cultured by replacing the serum with an excess of broth. Urine specimens were collected only in older children with lobar pneumonia and precipitin tests for soluble specific substances were done on the fresh samples. Infected exudates were examined microscopically and by culture and mouse inoculation. In the seventy-one fatal cases, forty-eight postmortem heart punctures, forty lung punctures and thirtyeight lumbar punctures were done on the ward, and the material obtained was cultured. At autopsy pieces of consolidated lung were studied either by the pathologists or pediatricians by means of stained films, cultures and mouse inoculation. Heart blood cultures were also taken.

The presence of protective antibodies in the serum was determined by injecting $0.2 \mathrm{cc}$. of each serum sample intraperitoneally into each of four white mice, five to thirty minutes before the infecting doses of pneumococci were injected. The infecting doses were in $0.5 \mathrm{cc}$. volumes in broth and consisted of $1 \mathrm{cc} . \times 10^{-5}$, $10^{-6}, 10^{-7}$ and $10^{-8}$ of a five to seven hour blood broth culture of a recent mouse passage strain of the appropriate pneumococcus. Virulence controls received the three highest dilutions and were the last mice inoculated in each experiment. Pour plates of the dilutions were then made; $1 \mathrm{cc} . \times 10^{-8}$ yielded two to six colonies. Between thirty to forty minutes elapsed between beginning the dilutions and the making of the pour plates. The Types I and II virulence controls invariably died within forty-eight hours. One Type III virulence control receiving $1 \mathrm{cc} . \times 10^{-8}$ survived. All other Type III controls died. All virulence controls were studied by stained films of the peritoneal exudate and culture. Except those mice which died during the night, all gave pure, bile soluble cultures of the appropriate pneumococci from the hearts' blood. Mice were observed seven days, the survivors scored, then discarded. Gram stains of the peritoneal exudate of all mice were examined, and a complete bacteriological examination was made of mice dying irregularly.

The presence of agglutinins was studied by means of the thread test. These were done by diluting the serum $1 / 2,1 / 4,1 / 8$ and $1 / 16$ in broth. One-half cubic centimeter volumes were placed in tubes $12 \times 100 \mathrm{~mm}$., and inoculated by a fine capillary pipette with two small drops of a six to eight hour blood broth culture of the appropriate pneumococcus recently shown to be virulent. Controls of the pneumococcus were set up for each experiment in homologous and heterologous "diagnostic" horse antipneumococcus serum diluted in broth to end of titre. The tubes were read after incubation overnight at $37^{\circ} \mathrm{C}$. Precipitin tests were done on urine in the usual way; on serum, by ring tests in capillary tubes with undiluted antipneumococcus serum. 
The strains of pneumococci used for the protection and the thread tests-one Type I, two Type II and two Type III pneumococci-were obtained from children in the series. The media used in the medical and pediatric laboratories were prepared in a central media room according to the same formula. The broth was a

TABLE 1

Bacteriological diagnosis

\begin{tabular}{|c|c|c|c|c|c|c|c|c|}
\hline & \multicolumn{5}{|c|}{ Type of pneumococcus } & \multirow{2}{*}{ 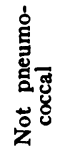 } & \multirow{2}{*}{ 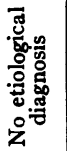 } & \multirow{2}{*}{ 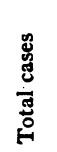 } \\
\hline & I & II & ical & III & IV & & & \\
\hline \multicolumn{9}{|l|}{ Children: } \\
\hline Lobar pneumonia (fixed type): & & & & & & & & \\
\hline Cases................. & 36 & 5 & 3 & 10 & 30 & 1 & 11 & 96 \\
\hline 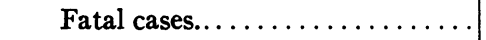 & 2 & & 1 & & 3 & & & 6 \\
\hline Mortality percentage........... & 5.5 & & 33 & & 10 & & & 6 \\
\hline Pneumonia (all types): & & & & & & & & \\
\hline Cases................ & 37 & 8 & 5 & 23 & 109 & 33 & 47 & 262 \\
\hline Fatal cases.............. & 2 & 2 & 1 & 2 & 34 & 19 & 5 & 65 \\
\hline Mortality percentage.......... & 5.4 & 25 & 20 & 9 & 31 & 57 & 11 & 25 \\
\hline \multicolumn{9}{|l|}{ Adults: } \\
\hline \multicolumn{9}{|l|}{ Lobar pneumonia (fixed type): } \\
\hline 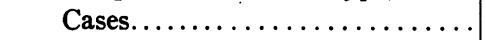 & 31 & 19 & 3 & 21 & 26 & 7 & 24 & 131 \\
\hline Fațal cases. .......... & 10 & 12 & 1 & 11 & 7 & 4 & 10 & 55 \\
\hline Mortality percentage.... & 32 & 63 & 33 & 52 & 28 & 57 & 41 & 42 \\
\hline \multicolumn{9}{|l|}{ Pneumonia (all types): } \\
\hline Cases............. & 32 & 20 & 6 & 32 & 48 & 28 & 60 & 226 \\
\hline Fatal cases......... & 11 & 13 & 1 & 14 & 13 & 14 & 28 & 94 \\
\hline Mortality percentage.... & 34 & 65 & 16 & 44 & 27 & 50 & 47 & 41 \\
\hline
\end{tabular}

Summary of Types $I, I I, I I$-atypical and III pneumococcus pneumonias Children Adults

Cases................................. $73 \quad 90$

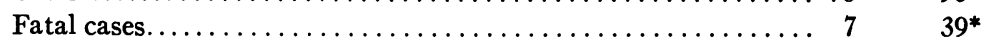

Mortality percentage $\ldots \ldots \ldots \ldots \ldots \ldots \ldots \ldots \ldots \ldots \ldots$

* Two of the 39 were postoperative pneumonias.

phosphate, infusion broth set to $\mathrm{pH} 7.8$ before being sterilized in the Arnold sterilizer. The agar was similarly prepared. Blood media was made by the addition of $\mathbf{5}$ per cent defibrinated rabbit's blood to the broth or agar. Blood agar plates were made daily, and used fresh without incubation for sterility.

The diagnostic antipneumococcus serum was obtained from the New York State Department of Health. Special high titre diagnostic serum obtained through the 
kindness of Dr. Mary B. Kirkbride was used to detect soluble specific substance in the urine and serum. Three children were treated with concentrated antipneumococcus Type I serum given by the Littauer Fund through the kindness of Dr. William H. Park.

\section{RESULTS}

\section{Incidence and mortality}

The cases are presented according to a clinical and a bacteriological classification in table 1. The outstanding point of divergence between the children and adults in the series is in the mortality rates for pneumonias due to the fixed type pneumococci. The mortality rate for

TABLE 2

Method of diagnosis in 34 children sick with pneumococcus Type I pneumonia

\begin{tabular}{|c|c|}
\hline \multicolumn{2}{|r|}{$\begin{array}{l}\text { Pneumococcus Type I } \\
\text { in } \\
\text { Throat-Swab-Mouse }\end{array}$} \\
\hline $\begin{array}{c}\text { Negative } \\
8 \\
\text { L }\end{array}$ & $\begin{array}{c}\text { Positive } \\
26\end{array}$ \\
\hline $\begin{array}{l}\text { Results of throat-swab- } \\
\quad \text { mouse } \\
\text { 1. Survived } \\
\text { 1. No pneumococci } \\
\text { 1. Pneumococcus III } \\
\text { 2. Pneumococcus IV } \\
\text { 3. Pneumococcus IV }\end{array}$ & $\begin{array}{l}\text { Method of Diagnosis } \\
\text { Positive pneumococcus Type'I blood culture } \\
\text { Positive pneumococcus Type I blood culture } \\
\text { Positive pneumococcus Type I blood culture } \\
\text { Positive pneumococcus Type I blood culture } \\
\text { Positive pneumococcus Type I protective bodies }\end{array}$ \\
\hline
\end{tabular}

adults is approximately four and a half times that of the children. By subtracting the number of cases of lobar pneumonia from the total number of cases in the series in table 1 it will be seen that there were few Type I or II pneumococci found in either the children or adults with bronchopneumonia. In the case of pneumococcus Type III, however, more than one-half of the cases in children and about onethird of those in adults were bronchopneumonia.

In many of the patients in this series Bacillus influenzae was recovered from the throat. This finding was especially common in those patients with bronchopneumonia due to pneumococcus Group IV, and in whom mixed infections with hemolytic streptococcus and staphylococcus in addition were demonstrated at autopsy. 
Table 2 has been prepared to show in part the limitations of the methods used in making a bacteriological diagnosis.

Table 2 shows that the final diagnosis in eight of thirty-four cases ${ }^{1}$ of pneumonia in children due to Type I pneumococcus was made by methods other than the throat-swab-mouse procedure. As the search for Type I protective bodies was carried out in only fourteen of the thirty cases tentatively diagnosed by the throat-swab-mouse method as Group IV pneumococcus lobar pneumonia and showed Type I antibodies in three of the fourteen, it is almost certain that a few fixed types were missed in this group of patients.

On the other hand, the correctness of the positive diagnosis, if a Type I or II pneumococcus was found in the throat, is checked by the development of appropriate protective bodies in thirteen of the fourteen Type I and II cases presented in figure 1 which were diagnosed by the throat-swab method. In this group the diagnosis failed to be corroborated only once; this one failure (case 9 , fig. 1) is subsequently discussed. There was a fatal case of encephalitis with a terminal bronchopneumonia in whom a pneumococcus Type I was recovered from the mouth during life, but not from pieces of the lungs nor from the postmortem heart-blood culture which gave a heavy growth of pneumococcus Group IV. A non-suppurative generalized encephalitis was found at autopsy. ${ }^{2}$

As to the possibility that fixed type pneumococci were overlooked in the fatal cases, it may be pointed out that there were no fatalities among the etiologically undiagnosed and the "non-pneumococcal" cases of lobar pneumonia in children. In the nineteen fatal cases of bronchopneumonia caused by bacteria other than pneumococci, and in the five etiologically undiagnosed cases, postmortem cultures of the hearts' blood were made in eighteen, blood cultures in the last two days of life in three and in the last three days in one; no blood cultures were made in two cases. None of these blood cultures contained pneumococci. In the thirty-four fatal cases of pneumonia due to Group IV pneumococci postmortem cultures of the hearts' blood were done in thirty-two and were negative for fixed type pneumococci.

\footnotetext{
${ }^{1}$ In three of the total thirty-seven cases of table 1 the diagnosis was made by blood culture and the mice were discarded without autopsy.

2 This case was classed as a Group IV pneumococcus pneumonia.
} 
These, together with the fatal cases due to fixed type pneumococci are presented in table 5 , and will be discussed later.

\section{Blood cultures}

The results of blood cultures in fifty-two children and seventy-four adults with Types I, II or III pneumococcus pneumonia are compared in table 3. Only cultures made during the active stage of the disease (i.e., before lysis or crisis) and postmortem are presented. One postmortem culture in an adult, and three in children are shown. There

TABLE 3

Blood cultures in Types I, II and III pneumococcus pneumonia in children and adults

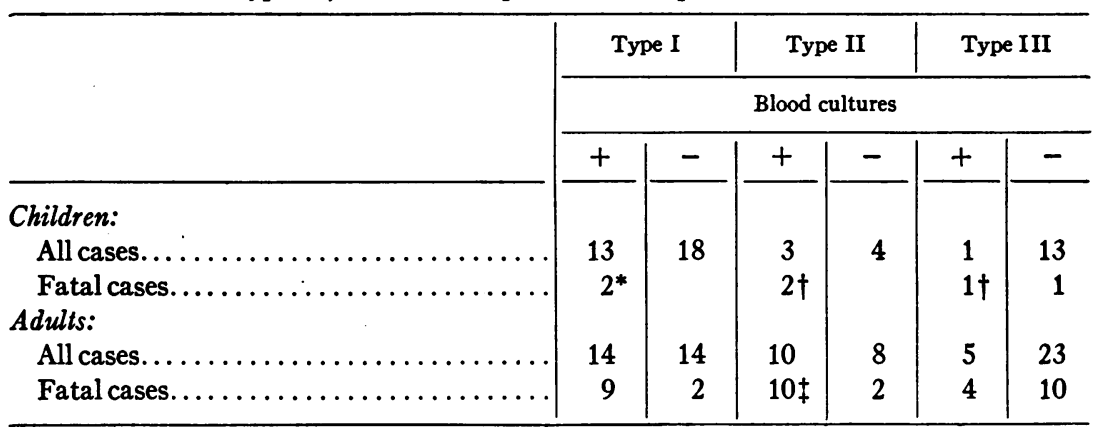

* One fatal Type I negative during life, positive postmortem.

† Only postmortem cultures taken in one Type II and the positive Type III.

$\ddagger$ One postmortem culture included.

139 cultures in 52 cases in children: 12 in 3 treated cases.

165 cultures in 74 cases in adults: 68 in 12 treated cases.

were three serum treated cases among the children with Type I infection and twelve among the adults; in these there was an average of four blood cultures per patient in the children and 5.5 per patient in the adults. In the untreated children the average number of blood cultures per patient was 2.5 and in the untreated adults 1.5 . The more frequent blood cultures in the untreated cases, the smaller number of serum treated cases and the greater number of postmortem cultures in the children would favor a greater incidence of positive cultures in the children than in the adults; but the figures show that the incidence was greater for each type of pneumococcus infection among the adults, being for the combined series 33 per cent positive cultures for the 
children, and 39 per cent for the adults. Among the children one of the fatal cases of Type I infection was negative during life and positive only after death; only postmortem cultures were taken in one of the fatal cases of Type II infection and in the fatal case of Type III infection showing a positive culture. Subtracting these three cases from the total the incidence of positive blood cultures during life for the children is fourteen, or 27 per cent, and subtracting similarly, the one adult case in which only postmortem blood cultures were taken, the incidence for adults is 38 per cent.

A hint of the prognostic significance of positive blood cultures in children may be obtained from the cases of Type I infection. As Lyon (18) observed, this finding is not so serious as in adults. However, nine of the thirteen children with positive blood cultures later developed a well marked empyema, only one dying with this complication. Empyema occurred in none of the eighteen patients with negative blood cultures. Six of the fourteen adults with positive blood cultures developed empyema, slight and detected only at autopsy in four. Of the fourteen adults with negative blood cultures empyema followed in one fatal treated case. Apparently in Type I infection a child with positive blood culture is likely to develop empyema with eventual recovery, whereas an adult is more likely to have a fatal termination. ${ }^{3}$

In children the few cases of Type II infection show approximately the same incidence of positive blood cultures as those with Type I infection but with a higher mortality; in adults with Type II infection the proportion of cases with positive cultures is higher and the outcome more often fatal than in patients with Type I infection. This reflects in part the peculiarities of pneumococcus Type II infection and possibly in part the effect of serum treatment.

The patients with atypical Type II infection are not presented in table 3. There were five such cases in children: one with slight but prolonged invasion of the blood, empyema and recovery; four with

3 This high incidence of Type I pneumococcus empyema in children was first pointed out by Lyon and is further corroborated by our findings in the ten cases of postpneumonic pneumococcus empyema admitted to the Hospital during this study. Eight had Type I pneumococcus empyema and recovered after operation They are not included in table 1 , nor have they been used in calculating mortality. 
negative cultures, of whom one was fatal on the tenth day of the disease with empyema found at autopsy. In this case culture of the heart's blood at autopsy was negative as were three blood cultures taken during life.

The comparatively slight blood stream invasiveness of pneumococcus Type III for adults is clearly indicated; for children it is still less invasive,- the only positive culture was obtained at postmortem. The association of pneumococcus Type III with focal lesions in children was observed in the occurrence of a profuse and prolonged mucoid

TABLE 4

Colonies per cubic centimeter of blood in pour plates in cases with positive blood cultures

\begin{tabular}{|c|c|c|c|c|c|c|c|c|c|}
\hline & \multicolumn{3}{|c|}{ Type I } & \multicolumn{3}{|c|}{ Type II } & \multicolumn{3}{|c|}{ Type III } \\
\hline & \multicolumn{9}{|c|}{ Colonies per cubic centimeter } \\
\hline & 0 to 10 & $\begin{array}{c}10 \text { to } \\
100\end{array}$ & $\begin{array}{c}100 \text { to } \\
1000\end{array}$ & 0 to 10 & $\begin{array}{c}10 \text { to } \\
100\end{array}$ & $\begin{array}{c}100 \text { to } \\
1000\end{array}$ & $\mid 0$ to 10 & $\begin{array}{c}10 \text { to } \\
100\end{array}$ & $\begin{array}{c}100 \text { to } \\
1000\end{array}$ \\
\hline $\begin{array}{l}\text { Children } . \ldots \ldots \ldots \ldots \ldots \ldots \ldots \ldots \\
\text { Adults. } . \ldots \ldots \ldots \ldots \ldots \ldots \ldots\end{array}$ & $\begin{array}{l}12 \\
11\end{array}$ & $1 *$ & $3 \dagger$ & $\begin{array}{l}2 \\
5 \ddagger\end{array}$ & & $\begin{array}{l}1^{*} \\
6\end{array}$ & 48 & & 1 \\
\hline
\end{tabular}

Children: 2 recovered cases serum treated.

Adults: 4 recovered and 9 fatal cases serum treated.

* Negative in pour plates during life. Positive plates at postmortem only.

$\dagger$ All three heavy blood cultures obtained before serum treatment.

$\ddagger$ In 4 of the 5 only one culture taken, in 2 three days before death and in 2 four days before death.

\& Three of the 4 cases were fatal. Blood cultures were taken on the day of death in 1 and the day before death in 2 .

' nasal or aural discharge containing large numbers of pneumococci in thirteen of the twenty-three Type III cases, but only one case of pneumococcus Type III pneumonia developed empyema, and this resolved after one aspiration. However, one of the ten postpneumonic empyemas mentioned in the preceding footnote was due to pneumococcus Type III.

The evidence so far shows that the pneumococcus invades the blood stream slightly less frequently in children than in adults. In table 4 , which shows the number of colonies developing per cubic centimeter of blood, a further quantitative difference appears. The record of Type I infection, unfortunately somewhat complicated by serum treatment, 
furnishes most cases for comparison. The only child with more than a few colonies per cubic centimeter of blood had negative blood cultures on the fourth and ninth day of disease but died on the fourteenth day with thirty colonies per cubic centimeter in the postmortem heart's blood. On the other hand, three of the adults had hundreds of colonies per cubic centimeter. These heavily positive cultures were all taken before serum treatment was begun. Six of the eleven adults with less than ten colonies per cubic centimeter died under serum treatment; without serum treatment almost surely most of them would

TABLE 5

Pneumococcus pneumonia in children (fatal cases)

Postmortem heart's blood cultures for pneumococcus

\begin{tabular}{|c|c|c|c|c|}
\hline \multirow{2}{*}{ Types } & \multirow{2}{*}{$\begin{array}{l}\text { Total } \\
\text { cases }\end{array}$} & \multirow{2}{*}{$\begin{array}{c}\text { Cases } \\
\text { cultured }\end{array}$} & \multicolumn{2}{|c|}{ Heart's blood cultures } \\
\hline & & & + & - \\
\hline Types I, II, II-Atypical, III. ....... . . . . . . & 7 & 6 & 3 & 3 \\
\hline Group IV $\ldots \ldots \ldots \ldots \ldots \ldots \ldots \ldots \ldots \ldots \ldots \ldots \ldots \ldots \ldots$ & 34 & 32 & 15 & 17 \\
\hline Totals............. & 41 & 38 & 18 & 20 \\
\hline
\end{tabular}

Colonies per cubic centimeter of postmortem heart's blood

\begin{tabular}{|c|c|c|c|c|c|}
\hline \multirow{2}{*}{ Types } & \multirow{2}{*}{$\begin{array}{c}\text { Positive } \\
\text { cases }\end{array}$} & \multirow{2}{*}{$\begin{array}{c}\text { Pour plates } \\
\text { not made }\end{array}$} & \multicolumn{3}{|c|}{ Colonies } \\
\hline & & & 0 to 10 & 10 to 100 & $\begin{array}{c}100 \text { to } 1000 \\
\text { or more }\end{array}$ \\
\hline $\begin{array}{l}\text { Types I, II, II-Atypical, III } \ldots \ldots \ldots \ldots \\
\text { Group IV } \ldots \ldots \ldots \ldots \ldots \ldots \ldots \ldots \ldots \ldots\end{array}$ & $\begin{array}{r}3 \\
15\end{array}$ & $\begin{array}{l}1 \\
6\end{array}$ & 6 & 1 & $\begin{array}{l}1 * \\
3 \dagger\end{array}$ \\
\hline
\end{tabular}

* Mixed infection with staphylococcus.

† Mixed infection with staphylococcus (1 case).

have developed a heavy infection of the blood. None of the fatal cases in children was serum treated.

In the Type II infections the difference between children and adults is clearer. The only child with heavily infected blood showed numerous colonies only in the postmortem culture; those cultures made repeatedly during life average less than two colonies per cubic centimeter, even within twenty-four hours of death. On the other hand, six of the adults had cultures showing hundreds to thousands of colonies, and of the other five, showing ten colonies or less, in two the last cultures were taken three days, and in two, four days before death. 
In patients with Type III infection, pour plates were not made in the only positive case among the children. The counts of plate colonies in the adults in this group show a much less severe invasion of the blood by pneumococcus Type III than occurred in the Type I and Type II infections.

In the seven fatal cases of pneumonia due to fixed type pneumococci shown in table 5, the postmortem heart-blood cultures were positive in three, (one Type I, one Type II and one Type III) and negative in three. In the remaining case dead on the sixth day in "status epilepticus" and not cultured postmortem, the blood cultures showed a few colonies of Type I pneumococci on the third and fourth days, none on the fifth. Of the three positive cases, pour plates were not made in one, a Type III infection; in the second, pour plates showed thirty colonies of pneumococcus Type I; and in the third, a pneumococcus Type II infection there were approximately 2000 colonies per cubic centimeter. However, in this last case, (the heavily positive Type II in table 4) many of the deep colonies were staphylococci, the child also having a staphylococcus pneumonia with staphylococcus pulmonary abscesses.

The postmortem heart-blood cultures of the Group IV cases are also presented in table 5. In view of Cooper's (19) studies of the Group IV pneumococci, and because the clinical picture associated with these organisms is often indistinguishable from that produced by Type I pneumococci, one feels that there may exist, among these Group IV organisms, strains essentially similar to the older fixed types so far as pathogenicity is concerned. This is not certain, however, and consequently all fatal cases whether of lobar or bronchopneumonia are presented. Cultures made in thirty-two of the thirty-four cases were positive in fifteen and negative in seventeen. In nine of the fifteen, pour plates were made; one had approximately 2000 colonies of both pneumococcus Group IV and staphylococcus; in the remaining six the plates showed one or two colonies or the cultures were positive in broth only.

Among the non-fatal cases due to Group IV pneumococci there were seven with positive blood cultures. The heaviest of these had thirty colonies per cubic centimeter. No others had more than ten, most were positive in broth only. The only positive pneumococcus blood cul- 
ture, not already mentioned, occurring in any child with any disease on the pediatric service during the period of the study was obtained from an infant with a facial cellulitis. The child died on the seventeenth day of life with pneumococcus Group IV, a staphylococcus, and B. coli in the blood culture.

\section{Soluble specific substance}

Another phenomenon which closely parallels positive blood cultures and prognosis in adults is the presence in the urine and in the serum of the soluble specific substance of Dochez and Avery (20).

Since satisfactory collection of urine is difficult in young children the presence of the soluble substance was sought in the serum of twelve cases of pneumonia due to Type I pneumococcus, in five due to Type II and in five due to Type III; collections were made during the active stage of, the disease and during convalescence; the examinations were negative in all cases. Three of these cases had positive blood cultures; one was fatal. The soluble substance was not often detected in the urine and then only in Type I cases, but a systematic study was not made in cases of infection due to organisms of the other types. Six non-fatal Type I cases in which soluble substance was detected in the urine, were followed daily for a considerable period. The results were positive during the active stage of the disease and during convalescence, as in adults; but in two the soluble substance increased coincidently with recovery and in the remaining four it was found in the same amounts during the active stage of the disease, crisis and lysis, and several days of convalescence.

\section{Development of type specific humoral antibodies}

Figure 1 shows the presence of protective bodies in the serum and of type specific agglutinins, as measured by the thread test, in one fatal and twenty recovered cases of pneumonia. One case from whom both pneumocqccus Type I and Type III were recovered from the throat, is presented twice. In the twelve cases of pneumonia where Type I pneumococcus was found and in the five due to Type II pneumococcus, protective bodies appeared in all but one. This exception is the case just mentioned, number 9 in the list of Type $I$ cases and number 5 in the list of Type III cases in which protective bodies against Type III 


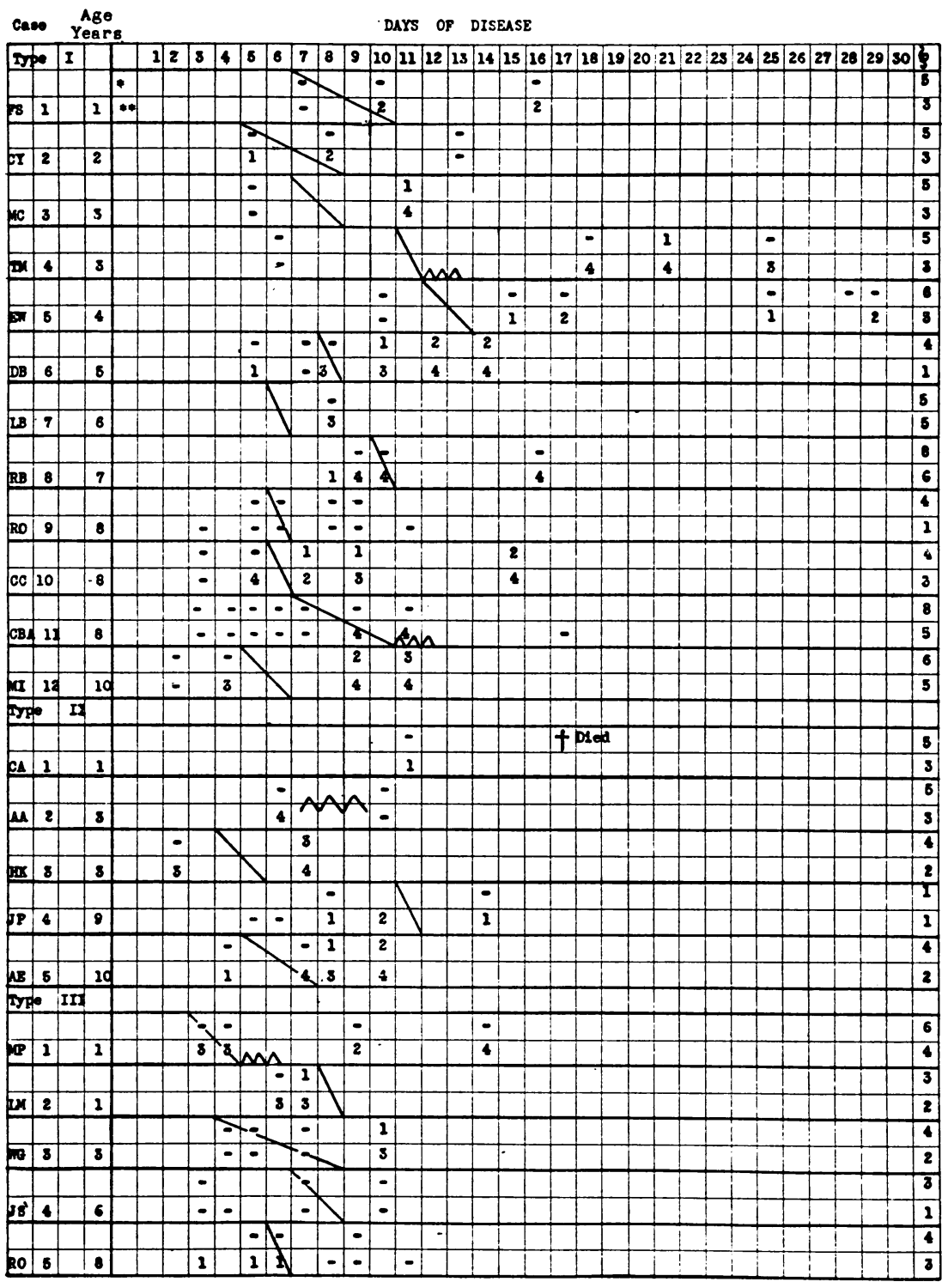

Fig. 1. Course of Agglutinins and Protective Bodies

* Upper lines $=$ thread tests. ${ }^{* *}$ Lower lines $=$ protection tests. $\searrow=$ fall of fever. All results: $1-4$, positive, - negative. $\S=$ Months between disease and time of tests. $M=$ fluctuating temperature over a long period.

Agglutination in dilutions Protection against M.L.D.

$\begin{array}{rlrrr}1 & 1-2 & & 1 & 1 \\ 2 & 1-4 & & 2 & 10 \\ 3 & 1-8 & & 3 & 100 \\ 4 & 1-16 & & 4 & 1000\end{array}$




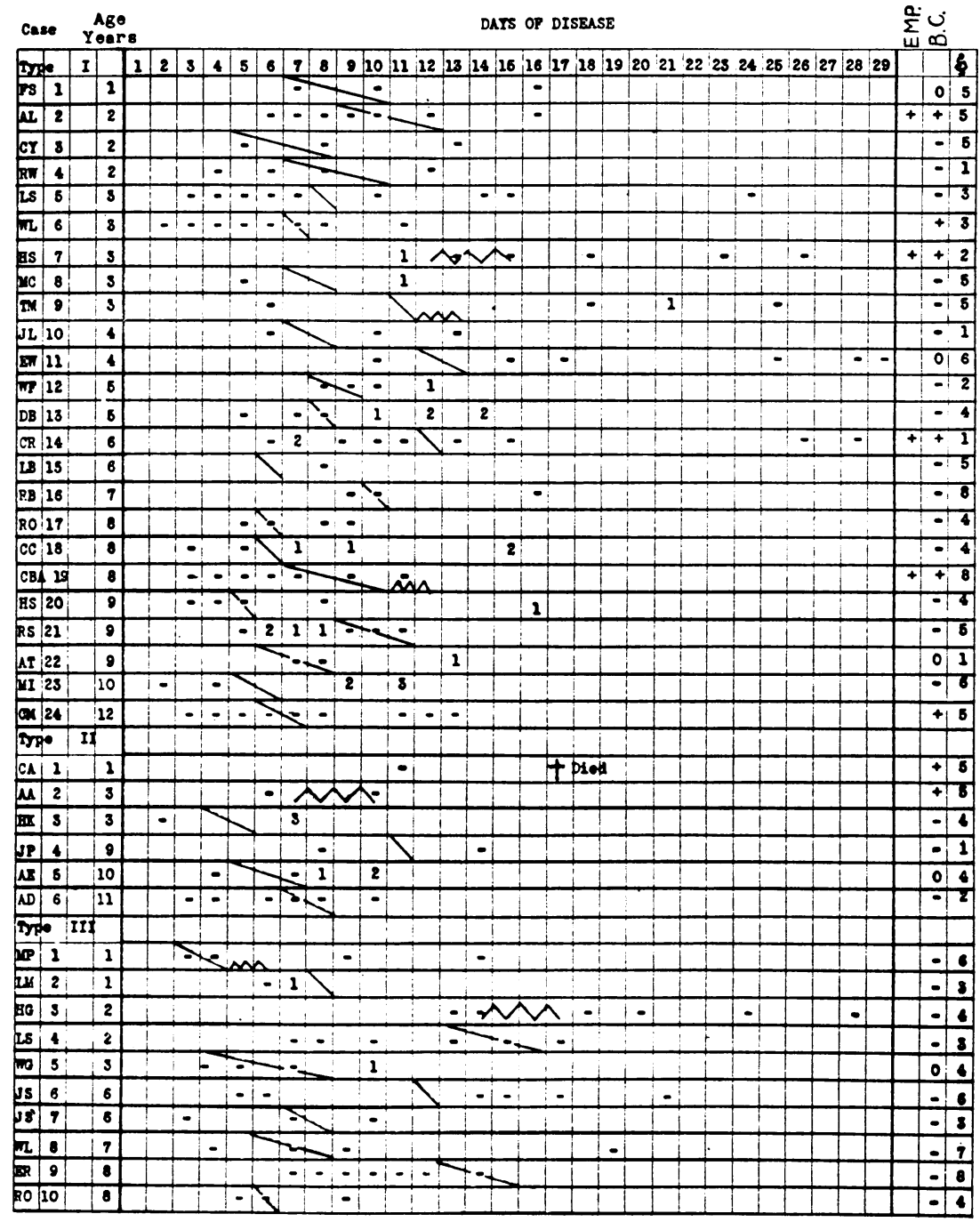

Fig. 2. Course of Agglutinins

1, 2, 3, 4 = agglutination in dilutions 1-2, 1-4, 1-8, 1-16 respectively. EMP. $=$ empyema. B. $\mathrm{C} .=$ blood culture. $\delta=$ months between disease and test. $+=$ positive or present. $\quad-=$ negative. $0=$ not done during active stage. Age is given in years. $\searrow=$ fall of fever. $M=$ fluctuating temperature over a long period. 
pneumococcus were produced but not against Type I. One may, therefore, presume that the Type I organism was not the etiological agent in causing the disease. Protective bodies against pneumococcus Type III were formed by three of the five Type III cases. The time of appearance of protective bodies corresponded fairly closely with recovery in all types. The fatal pneumococcus Type II pneumonia which had slight protection on the eleventh day died on the seventeenth with Type II pneumococci and staphylococci in the heart's blood and staphylococcus abscesses in the lungs.

The detection of agglutinins in cases of pneumonia was less regular, positive results being obtained in five of the Type I, two of the Type II and two of the Type III cases. Agglutination was, with one exception, not observed unless there was protection against 100 or 1000 M.I.D. The exception is number 10 of the Type I cases; on the seventh day there was protection against only 10 M.L.D.

There were thirteen samples of serum giving protection against 100 M.L.D., of which five gave positive and eight, negative thread tests; there were nineteen samples giving protection against 1000 M.L.D or more, nine of which gave positive and ten, negative thread tests.

In 1928, a series of cases was studied in the same way as those shown in figure 1; but the protection tests were done by a different technic, the serum samples being injected into the mice twelve to eighteen hours before the infecting dose of pneumococci. As suggested by Dr. Lord, ${ }^{4}$ this method was found to be unsatisfactory for detecting small amounts of antibody, and as the results with this technic were irregular they have been discarded. However, the thread tests were done the same way both years and they are shown in figure 2 combined with those previously shown in figure 1 .

One fatal and thirty-eight recovered cases are presented in figure 2. As in figure 1, one case is presented twice; it is number 17, of the Type I series and number 10 of the Type III. The cases are arranged according to type of pneumococcus and under each type, according to age. The results may be summarized in tabular form, positive or negative for the development of type specific agglutinins, as shown at top of page 638 .

${ }^{4}$ Personal communication. 


\begin{tabular}{c|c|c|c|c|c}
\hline \multicolumn{2}{c|}{ Type I (24 cases) } & \multicolumn{2}{|c}{ Type II (6 cases) } & \multicolumn{2}{c}{ Type III (10 cases) } \\
\hline+ & - & + & - & + \\
\hline 11 & 13 & 2 & 4 & 2 & 8 \\
\hline
\end{tabular}

The absence of detectable agglutinins in eight and low titre in three of the first eleven cases (four years or under) among the pneumonias due to pneumococcus Type $I$ is quite striking; a similar finding is shown for all the Type III cases. In neither of these groups was there agglutination in serum dilutions higher than one to two.

The presence or absence of positive blood cultures and the occurrence of empyema is noted in figure 2 to bring out the point that these features are not obviously related to the development of agglutinin. The duration of storage of serum samples is given in figures 1 and 2 . Three potent samples left over from 1928 were retested in 1929-a ten month's interval - and were found to be of the same titre each time.

In a series of twelve cases of pneumonia in children due to Type I pneumococcus and five due to Type III an attempt was made to follow the course of the common (non-type specific) pneumococcus agglutinin by means of thread tests using degraded avirulent pneumococci of homologous and heterologous origins as antigens. The tests were positive during the disease and in convalescence in all cases, but the changes in titre were too variable to be correlated in so small a series with any feature of the disease. Therefore, these results are not presented in detail.

\section{DISCUSSION}

The more important previous reports of the bacteriology of pneumonia in infants and children may be summarized as follows:

\begin{tabular}{|c|c|c|c|c|c|c|c|}
\hline Authors & Cases & $\begin{array}{l}\text { Lobar } \\
\text { pneu- } \\
\text { monia }\end{array}$ & $\begin{array}{l}\text { Fixed } \\
\text { types }\end{array}$ & $\underset{\text { IV }}{\text { Group }}$ & $\begin{array}{c}\text { Broncho- } \\
\text { pneumonia }\end{array}$ & $\begin{array}{l}\text { Fixed } \\
\text { types }\end{array}$ & $\underset{\text { IV }}{\text { Group }}$ \\
\hline & & & per cent & per cent & & per cent & per cent \\
\hline Wollstein and Benson (21)........ & $50^{*}$ & 11 & 45 & 36 & 36 & 19 & 56 \\
\hline Pisek and Pease (22)... & 63 & 31 & 68 & 23 & 32 & 25 & 38 \\
\hline Mitchell (23) . . . . . . . & 90 & 79 & 28 & 72 & 11 & 9 & 91 \\
\hline Lyon (18). . & 151 & 99 & 39 & 38 & 52 & 13 & 38 \\
\hline Webster $(24) \dagger \ldots$ & 137 & 120 & 49 & 41 & 17 & 12 & 53 \\
\hline Westlund (25). . . . . . & 103 & 69 & 26 & 61 & 34 & 18 & 53 \\
\hline
\end{tabular}

* Three cases of bronchitis.

$\dagger$ Patterson's figures for one hundred and forty-seven adults with lobar pneumonia in the same city: 57 per cent fixed types, 35 per cent Group IV. 
Lyon (18) was the first to emphasize the fact that the incidence of Type I pneumococci in lobar pneumonia in children approaches that found in adults. In his tabulation, fifteen of his twenty-nine cases of Type I lobar pneumonia were diagnosed only by other means than the throat-swab-mouse method, (blood culture, lung puncture and empyema fluid). It is thus obvious that many cases of fixed type pneumococcus infection may be missed in children.

Webster (24) working in the Children's Hospital of Melbourne tabulated his results with those of Patterson (26) working with adults in the Melbourne Hospital. Webster's study was made in 1922-1924 and Patterson's in 1920-1922. The tabulation upholds Lyon's contention that there is little difference in the etiology of lobar pneumonia in children and in adults with respect to the fixed type pneumococci. The truth of this statement becomes obvious if the second footnote to the above tabular summary is consulted, when it will be seen that there were 35 per cent of Group IV strains among the adults compared with 41 per cent among the children. It seems probable that at least some of the cases in children classified as due to Group IV pneumococci were in reality due to fixed types for reasons pointed out above.

The mortality in children with pneumonia due to fixed type and Group IV pneumococcus-diagnosed either as lobar or bronchopneumonia -is tabulated below and the mortality compared with that in adults where data have been given by the original authors.

\begin{tabular}{|c|c|c|c|c|c|c|c|c|}
\hline \multirow{3}{*}{ Authors } & \multicolumn{4}{|c|}{ Fixed types } & \multicolumn{4}{|c|}{ Group IV } \\
\hline & \multicolumn{2}{|c|}{ Children } & \multicolumn{2}{|c|}{ Adults } & \multicolumn{2}{|c|}{ Children } & \multicolumn{2}{|c|}{ Adults } \\
\hline & $\begin{array}{l}\text { Total } \\
\text { cases }\end{array}$ & $\begin{array}{l}\text { Mor- } \\
\text { tality }\end{array}$ & $\begin{array}{l}\text { Total } \\
\text { cases }\end{array}$ & $\begin{array}{l}\text { Mor- } \\
\text { tality }\end{array}$ & $\begin{array}{l}\text { Total } \\
\text { cases }\end{array}$ & $\begin{array}{l}\text { Mor- } \\
\text { tality }\end{array}$ & $\begin{array}{l}\text { Total } \\
\text { cases }\end{array}$ & $\begin{array}{l}\text { Mor- } \\
\text { tality }\end{array}$ \\
\hline & & $\begin{array}{l}\text { per } \\
\text { cent }\end{array}$ & & $\begin{array}{l}\text { per } \\
\text { cent }\end{array}$ & & $\begin{array}{l}\text { per } \\
\text { cent }\end{array}$ & . & $\begin{array}{l}\text { per } \\
\text { cent }\end{array}$ \\
\hline Wollstein and Benson (21). & 12 & 58 & & & 28 & 43 & & $\therefore$ \\
\hline Pisek and Pease $(22)^{*} \ldots \ldots$ & 29 & 24 & 57 & 38 & 19 & 21 & 15 . & 6 \\
\hline Mitchell (23)........... & 23 & 13 & & & 67 & 16 & & \\
\hline Lyon (18) .............. & 46 & 13 & 99 & 37 & 58 & 17 & 37 & 11 \\
\hline Webster (24)........... & 45 & 2 & 84 & 29 & 49 & 4 & 57 & 37 \\
\hline Present study. . & 73 & 10 & 90 & 43 & 109 & 31 & 48 & 27. \\
\hline
\end{tabular}

* Pisek and Pease obtained their figures for adults from Cole. 
With respect to pneumonia due to the fixed types it seems clear that the mortality is much lower in children than in adults. The high fatality in children found by Wollstein and Benson and by Pisek and Pease has not been seen in the more extended studies of later investigators. The first named authors were dealing with children one month to four and a half years of age, most of them being eight to eighteen months. Pisek and Pease found as indicated, a lower mortality than Cole (27) observed working with adults about the same time and place. The difference in mortality in Lyon's series is marked; this difference is especially marked in Webster's series where fifteen recovered cases are omitted because the children received serum treatment. If these are included his figures show only one death in sixty fixed type cases. The difference is also well marked in the authors' series. In the case of pneumonia due to Group IV pneumococci, on the other hand, no such difference is apparent.

There are certain obvious difficulties in these comparisons, even though the mortality in adults and children has been compared in the same place and season. In the first place it is impossible to know whether equal proportions of patients of the same kind are admitted to the hospitals, so that the matter of sampling may not be well controlled. In the second place the number of cases reported is still relatively small. Thirdly, it might be objected that there exist variations among members of the same types of pneumococci, even though up to the present time bacterial variation in this sense has not been shown to occur in the pneumococci found during the acute stage of pneumonia. To those who regard the differences in pneumococcus lobar pneumonia in children and adults as a problem in comparative immunology the issue resides in the propriety of maintaining that the same parasite causes a mild disease in children and a severe one in adults. In spite of the difficulties noted above the trend would nevertheless seem definite enough toward this hypothesis.

The chief offensive mechanism of the pneumococcus for adults. whatever it may be, seems to be measured by the capacity of the organism to invade tissue as is shown by spreading pulmonary lesions and particularly by its capacity to invade the blood as is shown by the frequent occurrence of heavily positive blood cultures. This was demonstrated by Dochez (28) in a study of thirty-seven cases of pneu- 
mococcus pneumonia. There were eighteen fatal cases; only four of these died with negative blood cultures. One of the four died on the forty-fifth day of the disease with nephritis and should be excluded from consideration; the other three died with spreading pulmonary lesions. Of the remaining fourteen who had positive blood cultures, all died with increasingly heavy septicemia. In twelve cases pour plates were made just before or just after death. These showed more than ten colonies per cubic centimeter of blood in one case, more than 100 colonies per cubic centimeter in one case, more than 1000 colonies per cubic centimeter in four cases, more than 10,000 in four and more than 50,000 in two.

While it is perhaps not pertinent to discuss in this place infections due to Group IV pneumococci, yet almost surely there are members of this group capable of producing a lobar pneumonia indistinguishable from that caused by the fixed types. Accordingly, for the sake of completeness and to add more cases, attention is again called to table 5 to show the rarity of heavily positive cultures of the postmortem heart's blood in children with all varieties of pneumococcus pneumonia.

The development of type specific antibodies in pneumococcus pneumonia as measured by agglutinins by Lister (9), Clough (10), Chickering (11), Cole (12) and Blake (13), or by protective bodies by Dochez (8), Baldwin and Rhoades (14) and Baldwin and Cecil (15), or by specific pneumococcidal bodies by Robertson and his collaborators (16), has been clearly shown to be intimately connected with recovery in adults. Indeed, until Tillet's (29) studies of pneumococcus Type III infection in rabbits, the development of these antibodies was considered to be probably the chief mechanism of defence against invasion by the pneumococcus.

Our own studies with protection tests in children, unfortunately practically limited to recovered cases, show that protective bodies develop at the time of recovery in them as regularly as in adults. Thus, so far as the mechanism of recovery is concerned the development of these bodies must also be given an important place in children. Our results with the thread tests, on the other hand, shows that less type specific antibody is formed by children than by adults. If the reverse had been true it might have explained the more frequent recovery seen in children. 
A possible criticism of our results exists in our not having done the tests for type specific antibodies immediately after a series of serum samples had been collected. Indeed the implication, in cases number 8 and 11 in figure 1, is quite strong that agglutinin had deteriorated in the months of storage. However, Poole and Sharp (30), who followed the development of type specific agglutinins in adults with pneumococcus pneumonia by means of thread tests carried out after the year's series of bleedings had been collected and with precisely the same technic, found that all recovered cases developed agglutinins. But irrespective of this question of deterioration and other possible variations in technic which might conceivably invalidate the comparison of our results with children with those of other workers with adults, it is, nevertheless, clear enough that children four years of age and under make definitely less type specific antibody than those who are older. It is also noteworthy, though perhaps of different significance, that in children with Type III pneumococcus pneumonia a lower antibody titre is found than in those with Type I or Type II infections. This relation between age and antibody formation is not surprising since Halber, Hirszfeld and Mayzner (31) found children did not begin to make any considerable amounts of typhoid agglutinin until they had passed the first year of life.

In discussing the relation of type specific antibody to the termination of pneumococcus pneumonia and while considering the relation of age to the development of type specific antibody, it is worth noting that we have seen three children (two during the study and one since) under four years of age finally recover from pneumonia and subsequent empyema (two Type I cases and an atypical Type II case), who had positive blood cultures until after the nineteenth, twenty-fifth and seventy-eighth day of disease respectively, even though the empyema cavity was draining. In adults one occasionally sees prolonged pneumococcemia but then with endocarditis and death.

The excretion of soluble specific substance might conceivably point to another mechanism of defense. However, all tests on serum samples for this substance were negative and while the results of studies on the urine were suggestive, total urine samples were not obtained and accordingly the evaluation of this part of the study is not possible.

As pointed out above the pneumococcus appears to invade the blood 
stream of children less frequently and less intensively than is the case in adults. At first glance this might seem to indicate something peculiar to the pneumococcus, because other acute respiratory pathogens are more invasive in children than in adults and the tubercle bacillus also is credited with being more invasive in children.

Thus, among the two hundred and sixty-two cases of pneumonia in children listed in table 1 there were four, (exclusive of cases of influenzal meningitis) with positive blood cultures showing Bacillus influenzae -in pure culture in two cases and mixed with pneumococcus Group IV in two. By contrast, in the two hundred and twenty-nine adult cases there were no blood cultures positive for this organism; perhaps, however, the comparison is not quite fair as there were more terminal and postoperative cases of pneumonia among them. In the war time epidemics of pneumonia where Bacillus influenzae was generally distributed, this organism was infrequently found in the heart's blood even by those workers who regularly found it in lungs and bronchi. Thus Opie, Blake, Small and Rivers (32) in two hundred and eighteen postmortem heart-blood cultures found it only once. Jordan (33) collected from the literature a thousand instances of blood cultures made during life in cases of influenza and influenzal pneumonia. The influenza bacillus was found but once. This notion of the relation of age of host to invasion by Bacillus influenzae is supported by Liston's (33a) report of a postmortem bacteriological study of forty cases of bronchopneumonia in children. He found Bacillus influenzae once in pure culture from the heart's blood and five times in association with other organisms. It is also in keeping with Rivers' (34) study of influenzal meningitis, which he found to be practically limited to infants and young children and in which positive blood cultures occurred in eight of his last eleven cases.

There were fifteen fatal cases of hemolytic streptococcus pneumonia among children in the series here reported, seven considered bronchogenetic, on purely clinical, and eight on clinical and pathological grounds. Among them blood cultures were positive in six of twelve studied during life and in six of nine in whom postmortem heart-blood cultures were made. In two of the six positive during life there were hundreds of colonies of hemolytic streptococci in the pour plates. There were only five fatal cases of hemolytic streptococcus pneumonia 
among the adults, one of three had positive blood cultures during life and four of five at postmortem. It is difficult to get cases enough to compare fairly the susceptibility of children and adults to blood stream invasion in hemolytic streptococcus pneumonia. By turning to the highly fatal postmeasles and postinfluenzal epidemics of the United States Army cantonments of 1918-1919, the high mortality of the epidemic should favor finding greater invasion among the adults. Unfortunately most of the material is limited to postmortem examinations. In Opie, Blake, Small and Rivers' (32) series of eighty-three autopsies in which Streptococcus hemolyticus was found in the bronchi or lungs it was found in the heart's blood in seventy-three and not found in ten. In MacCallum's (35) series it was found in twenty-three of twenty-five cases in the heart's blood, but in his nine cases studied during life it was found only twice. Our own rather limited past experience with quantitative blood cultures during life in adults and our current experience with children is that the latter more frequently furnish heavily positive blood cultures than the former during the course of hemolytic streptococcus pneumonia.

In the twelve fatal cases of bronchogenetic staphylococcus pneumonia in children in table 1, four of nine studied had blood cultures positive for staphylococcus during life and seven of eleven had positive heart's blood postmortem. There were only two cases of bronchogenetic staphylococcus pneumonia in the adult series. One showed a negative postmortem heart-blood culture and the other had negative blood cultures the day of death and was not examined at postmortem. The number of cases is too small for comparison, but consideration of the age incidence of staphylococcus osteomyelitis indicates the probability that the staphylococcus is more invasive for children than adults.

It would be desirable if infections with these common respiratory pathogens other than the pneumococcus could be analyzed more extensively with respect to recovered and fatal cases in children and in adults. However, at present there is insufficient data to do this.

The invasiveness of pneumococcus for small laboratory animals is impressive. When Welch (36), Wadsworth (37), Kirkbride (38) and others attempted to produce pneumococcus pneumonia in rabbits they found that the normal animal developed not pneumonia, but a 
rapidly fatal septicemia. In the partially immunized animal, however, injections of the pneumococcus resulted in a pneumonia. Stillman and Branch (39) have recorded similar differences in response between normal and partially immunized mice. Blake and Cecil (40) described an experimental pneumococcus Type I pneumonia in monkeys almost identical with the natural disease as seen in man. In certain respects, however, the picture differed somewhat from that in man. The animals seemed less prostrated early in the disease, blood stream invasion was heavier, and the fatality was greater. However, in monkeys that were partially immunized to pneumococcus and later experimentally infected, a pneumococcemia was then observed which was more comparable to that seen in the natural disease in man and the fatality was diminished. Cecil and Blake (41) also took the opportunity of studying a natural epidemic which occurred in one lot of animals and observed that monkeys could develop a spontaneous lobar pneumonia, in this instance due to pneumococci of Group IV.

In view of the foregoing observations the capacity for localizing the pneumococcus sufficiently to produce a lobar pneumonia is generally taken to show the presence of a partial immunity. The mechanism of man's acquisition of this property and the possible accompanying reactivities are still matters for speculation. Consequently, if one were asked to compare the relationship between experimental studies in pneumococcus infection in animals and studies of the disease in adult man it would be wisest to seek for more informa tion first. However, with the material at hand the following highly problematical schema might be helpful:

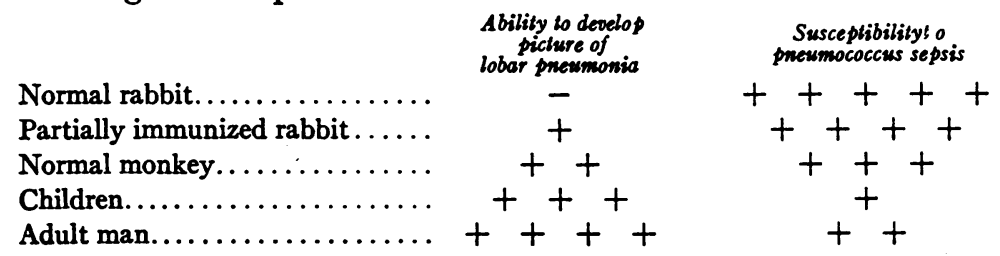

It is obvious at once that as regards susceptibility to pneumococcus sepsis the data concerning children and adults do not fall into regular sequence, i.e., the children do not evidence greater susceptiblity to pneumococcus sepsis than do adults. The schema, however, so far as 
human beings are concerned is developed from data in which recovered and fatal cases of pneumonia are considered together. If now, on further analysis, only recovered cases of pneumococcus pneumonia are considered, a striking change will be observed. By combining Lyon's figures with our own it is possible to collect thirty-seven non-fatal cases of pneumococcus Type I pneumonia in children who were untreated and in whom blood cultures were taken. Of these, fourteen or 38 per cent had positive blood cultures. However, clinical experience with adults would indicate with respect to the incidence of positive blood cultures that (1) it is less than 38 per cent in non-fatal cases; (2) it is less in non-fatal cases due to pneumococcus Type II than in those due to pneumococcus Type I; and (3) it is less in untreated cases than in those treated with serum. This is shown in the following figures.

\begin{tabular}{|c|c|c|c|c|}
\hline Author & Type & Cases & Treated & $\begin{array}{l}\text { Incidence } \\
\text { of positive } \\
\text { cultures }\end{array}$ \\
\hline \multirow{3}{*}{ Cole et al.. } & & & & per cent \\
\hline & I & 129 & Yes & 29 \\
\hline & II & 103 & Few cases & 13 \\
\hline \multirow{2}{*}{ Cecil et al.. } & I & 30 & Yes & 17 \\
\hline & II & 8 & No & 13 \\
\hline \multirow{4}{*}{ Park et al.. } & I & 69 & No & 12 \\
\hline & I & 90 & Yes & 20 \\
\hline & II & 40 & No & 5 \\
\hline & II & 43 & Yes & 16 \\
\hline
\end{tabular}

Thus, if only recovered cases are considered as regards pneumococcus sepsis the relative positions of children and of adults in the schema are really in regular sequence of decreasing susceptibility and conform with similar facts concerning the other infections mentioned above. It becomes obvious that it is the fatal cases in adults for which some new factor must be considered. To demonstrate these points figure 3 is presented. The inference which may be drawn from the facts represented in the figure is that a sharp distinction should be made between a mere positive blood culture and the increasingly heavy septicemia which characterizes most fatal cases in adults. The former may measure the pathogenicity of pneumococcus for man but the latter appears 
to measure a unique phenomenon at present recognized only in adult man, and representing a response in him different from that which he exhibits in childhood. In elaborating this point of view recourse to analogies may be permitted until the mechanism of the breaking of defense against pneumococcus sepsis has been demonstrated.

Countless workers have applied the principles of Koch's tuberculin reaction to the study of tuberculosis. In this country Baldwin (42), Krause (43), Soper (44) and Rich (45) are especially known for such investigations, which have shown among other things, that there is an association between tissue invasion and local tissue damage and

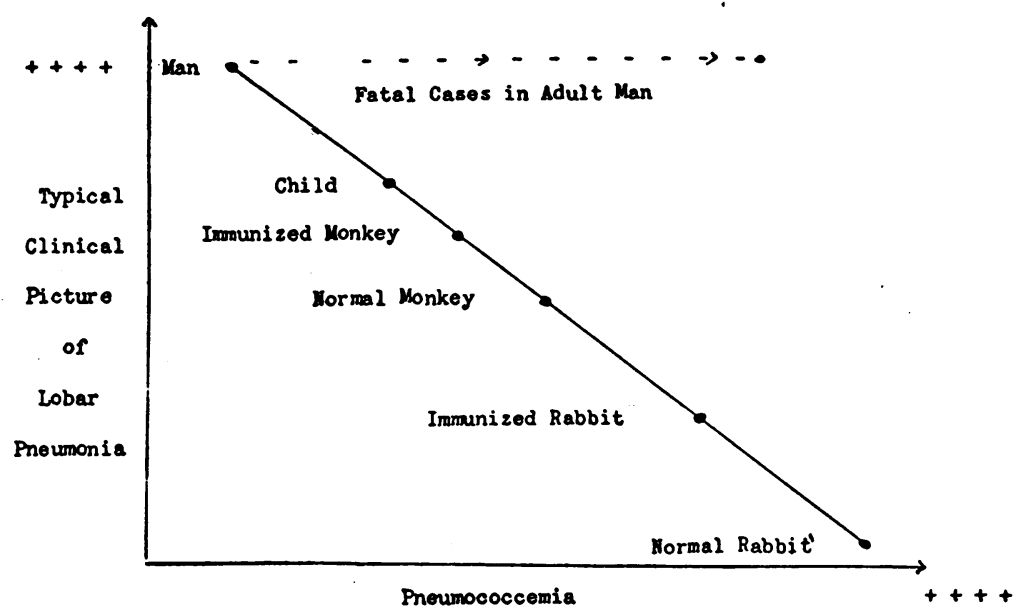

Fig. 3. Diagram of Ability to Develop Typical Picture of Clinical Lobar

Pneumonia Compared with Susceptibinity to Pneumococcemia in Man, ChInd, Monkey and Rabbit

immunity to the tubercle bacillus. Besides this Opie (46) has been interested in the general problem of the response of animals to the repeated injections of antigen, and at one place in a series of studies on the Arthus phenomenon after mentioning the tuberculin, luetin and typhoidin reactions he says, "In these and other infectious diseases there is the paradoxical association of increased resistance to the microörganism and heightened local susceptiblity to substances obtainable from it." It seems to be a widely applicable generalization that the acquisition of an immunity temporarily effective for the defense of the whole animal is often accompanied by the acquisition of a 
susceptibility so that the locally defending tissues-insusceptible before the acquisition - may be damaged or killed by the products of the infecting bacteria. Moreover Dochez and Sherman (47) and Dochez and Stevens (48) have shown for animals and Cooke (49) has shown for man that a neutralizable susceptibility to toxins of hemolytic streptococci may be an acquired susceptibility.

In pneumococcus infections this noxious agent may not be a toxin in the conventional sense. In fact, the number of investigators who have had negative results in attempts to find such a substance, shows that, if it exists, it will probably not be demonstrated by conventional methods alone. In this connection, however, it is interesting that Parker (50) recently described a new "pneumococcus toxin" which presumably acts on the guinea pig's ${ }^{5}$ defense mechanism against the invasive property of the pneumococcus, inasmuch as she demonstrated the toxin by means of a system containing sublethal doses of the toxin and normally sublethal doses of live pneumococci. The animals killed by the mixture showed great numbers of pneumococci in the heart's blood, whereas those protected with the antitoxin showed sterile heartblood cultures when sacrificed after survival.

\section{SUMMARY}

The evidence shows that children recover from pneumonia due to the fixed types of pneumococcus relatively more frequently than adults. The difference in fatality could be on the basis of many changes incident to age. However, not having data on other possible factors and being concerned with immunological responses, we have regarded the problem with a narrow view and have sought to emphasize special features.

Associated with the lower mortality in children is a less frequent and less intense degree of blood stream invasion that cannot be explained by the observed production of type specific humoral antibodies. This less marked invasion is at variance with the experience with other common respiratory pathogens which are more invasive in childhood than in adult life, but this inconsistency is not seen if recovered cases alone are considered. Attention thus redirected becomes focused

- It should be noted that Smith (51) and others (52) have found the guinea pig is naturally susceptible to pneumococcus infections. 
on the fatal cases in adults, which may then be observed to be the group that presents the anomalous feature.

In explanation it may be suggested either that children possess a mechanism of defense different (in a positive sense) from that of adults and perhaps analogous to the defense of the rabbit against pneumococcus Type III described by Tillet (29), or that adults acquire a susceptibility to pneumococcus substances, which then could act against the defense mechanism by which heavy blood stream invasion is prevented. The two hypotheses are not mutually exclusive. Both factors, as well as others, may be operative, but practicing physicians have always been impressed with the appearance of toxemia shown by adults with pneumococcus pneumonia, and the time relation of the premortal increasing bacteriemia to the course of the disease in fatal cases seems to indicate a suddenly breaking defense. As susceptibility to various products of other pathogens have been shown to be acquired during life, we are inclined to emphasize the latter factor.

\section{CONCLUSIONS}

1. Fixed type pneumococci cause a large proportion of the cases of lobar pneumonia in childhood, and are uncommon in cases of bronchopneumonia during this period.

2. Pneumonia due to Types I and III pneumococci has a much lower mortality in children than in adults.

3. Heavy blood stream invasion in children with pneumococcus pneumonia is rare.

4. Protective bodies appear in children with pneumococcus pneumonia, as in adults, about the time of recovery. Type specific agglutinins similarly appear in children, but their appearance is less frequent than in adults, and apparently less frequent in younger than in older children.

5. The marked pneumococcemia seen in fatal cases of lobar pneumonia in adult man suggests the presence of some special reactivity on his part towards the pneumococcus different from that possessed by him in childhood.

We take especial pleasure in thanking Dr. Francis G. Blake and his staff for the bacteriological studies on the cases of pneumonia in adults. 


\section{BIBLIOGRAPHY}

1. Neufeld, F., and Händel, Arb. k. Gsndhtsamte., 1910, xxxiv, 293. Weitere untersuchung über Pneumokokken Heilsera. III. Mitteilung. Uber Vorkommen und Bedeutung atypischer Varietäten des Pneumokokkus.

2. Dochez, A. R., and Gillespie, L. J., J. Am. Med. Assoc., 1913, lxi, 727. A Biologic Classification of Pneumococci by Means of Immunity Reactions.

3. Avery, O. T., Chickering, H. T., Cole, R., and Dochez, A. R., Monogr. Rock. Inst. Med. Res., 1917, No. 7. Acute Lobar Pneumonia. Prevention and Serum Treatment.

4. Cecil, R. L., Baldwin, H. S., and Larsen, N. P., Arch. Int. Med., 1927, xl, 253. Lobar Pneumonia; a Clinical and Bacteriologic Study of 2000 Typed Cases.

5. Park, W. H., Bullowa, J. G. M., and Rosenblüth, M. B., J. Am. Med. Assoc., 1928, xci, 1503. The Treatment of Lobar Pneumonia with Refined Specific Antibacterial Sera.

6. Cole, R., De Lamar Lectures, The Williams and Wilkins Co., Baltimore, 1928. Acute Pulmonary Infections.

7. Stillman, E. G., J. Exper. Med., 1916, xxiv, 651. A Contribution to the Epidemiology of Lobar Pneumonia. J. Exper. Med., 1917, xxvi, 513. Further Studies on the Epidemiology of Lobar Pneumonia.

8. Dochez, A. R., J. Exper. Med., 1912, xvi, 665. The Presence of Protective Substances in Human Serum During Lobar Pneumonia.

9. Lister, F. S., South African Inst. Med. Res. Pub., Johannesburg, 1913, Memoir 3. Specific Serological Reactions With Pneumococci From Different Sources.

10. Clough, P. W., Bull. Johns Hopkins Hosp., 1913, xxiv, 295. The Development of Antibodies in the Serum of Patients Recovering From Acute Lobar Pneumonia. Bull. Johns Hopkins Hosp., 1919, xxx, 167. Phagocytosis and Agglutination in the Serum in Acute Lobar Pneumonia; the Specificity of These Reactions and the Regularity of Their Occurrence.

11. Chickering, H. T., J. Exper. Med., 1914, xx, 599. Agglutination Phenomena in Lobar Pneumonia.

12 Cole, Rufus, J. Exper. Med., 1917, xxvi, 453. The Neutralization of Antipneumococcus Immune Bodies by Infected Exudates and Sera.

13. Blake, F. G., Arch. Int. Med., 1918, xxi, 779. Antigen-Antibody Balance in Lobar Pneumonia.

14. Baldwin, H. S., and Rhoades, D. R., Bull. Hyg. Lab., Public Health Service, 1925, No. 141, p. 43. Studies on Pneumococcus Immunity. VII. The Protective Substance in Pneumococcus Pneumonia with Special Reference to Bacteriemia and Specific Treatment.

15. Baldwin, H. S., and Cecil, R. L., J. Am. Med. Assoc., 1926, lxxxvii, 1709. The Rationale of Specific Therapy in Pneumococcus Pneumonia. 
16. Sia, R. H. P., Robertson, O. H., and Woo, S. T., J. Exper. Med., 1928, xlviii, 513. A Study of the Mechanism of Recovery from Lobar Pneumonia.

17. Blake, F. G., Presented Before Section on Medicine, N. Y. Acad. Med., 1929. Observations on Type III Pneumococcus Pneumonia.

18. Lyon, A. B., Am. J. Dis. Child., 1922, xxiii, 73. Bacteriologic Studies of One Hundred and Sixty-five Cases of Pneumonia and Postpneumonia Empyema in Infants and Children.

19. Cooper, G., Edwards, M., and Rosenstein, C., J. Exper. Med., 1929, xlix, 461. The Separation of Types Among the Pneumococci Hitherto Called Group IV and Development of Therapeutic Serums for These Types.

20. Dochez, A.' R., and Avery, O. T., J. Exper. Med., 1917, xxvi, 477. The Elaboration of Specific Soluble Substance by Pneumococcus During Growth.

21. Wollstein, M., and Benson, A. W., Am. J. Dis. Child., 1916, xii, 254. Types of Pneumococcus Found in the Pneumonias of Infants and Young Children.

22. Pisek, G. R., and Pease, M. C., Am. J. Med. Sci., 1916, cli, 14. A Preliminary Report on Pneumonia in Children with Special Reference to its Epidemiology.

23. Mitchell, A. G., Penn. Med. J., 1917, xx, 343. The Types of Pneumococci in Infants and Children.

24. Webster, R., M. J. Australia, 1924, xi, pt. 1, 129. A Study of the Incidence of the Various Serological Types of Pneumococci in Pneumonia of Childhood: Observations on Serum Therapy.

25. Westlund, R. E., J. Infect. Dis., 1926, xxxviii, 514. The Incidence of Pneumococcus Types in Pneumonias of Children.

26. Patterson, S. W., M. J. Australia, 1922, ix, pt. 2, 115. The Prognosis of Lobar Pneumonia and the Influence of Serum in Treatment.

27. Cole, R., Arch. Int. Med., 1914, xiv, 56. Pneumococcus Infection and Lobar Pneumonia.

28. Dochez, A. R., J. Exper. Med., 1912, xvi, 680. The Occurrence and Virulence of Pneumococci in the Circulating Blood During Lobar Pneumonia and the Susceptibility of Pneumococcus Strains to Univalent Antipneumococcus Serum.

29. Tillet, W. S., J. Exper. Med., 1927, xlvi, 343. Studies on Immunity to Pneumococcus Mucosus (Type III). III. Increased Resistance to Type III Infection Induced in Rabbits by Immunization with $\mathbf{R}$ and $\mathbf{S}$ Forms of Pneumococcus. J. Exper. Med., 1928, xlviii, 791. Active and Passive Immunity to Pneumococcus Infection Induced in Rabbits by Immunization with R Pneumococci.

30. Poole, A. K., and Sharp, E. A., Unpublished Experiments. Course of Common and Type Specific Antibodies in Pneumococcus Pneumonia.

31. Halber, W., Hirszfeld, H., and Mayzner, M., Ztschr. f. Immunitätsforsch. u. Exper. Therap., 1927, liii, 391. Untersuchungen über die Antikörperentstehung bei Kindern im zusammenhang mit dem Alter. 
32. Opie, E. L., Blake, F. G., Small, J. C., and Rivers, T. M., Epidemic Respiratory Disease. C. V. Mosby Co., St. Louis, 1921.

33. Jordan, E. O., Epidemic Influenza. Am. Med. Assoc. Chicago, 1927.

33a. Liston, W. Glen, Arch. Dis. Child., 1929, iv, 283. Note on the Bacteriology of Bronchopneumonia of Children.

34. Rivers, T. M., Am. J. Dis. Childhood, 1922, xxiv, 102. Influenzal Meningitis.

35. MacCallum, W. G., Monogr. Rock. Inst. Med. Res., 1919. No. 10. The Pathology of the Pneumonia in the United States Among Camps During the Winter of 1917-18.

36. Welch, W. H., Bull. Johns Hopkins Hosp., 1892, iii, 125. The Micrococcus Lanceolatus, with Especial Reference to the Etiology of Acute Lobar Pneumonia.

37. Wadsworth, A., Am. J. Med. Sci., 1904, cxxvii, 851. Experimental Studies on the Etiology of Acute Pneumonitis. J. Exper. Med., 1912, xvi, 54. First paper. Studies on Pneumococcus Infection in Animals. J. Exper. Med., 1912, xvi, 78. Second paper. Action of Immune Sera on Pneumococcus Infection.

38. Kirkbride, M. B., J. Exper. Med. 1915, xxi, 605. A Study of the Effect of Sensitization on the Development of theLesions of Experimental Pneumonia in the Rabbit.

39. Stillman, E. G., and Branch, A., J. Exper. Med., 1930, li, $275 . \quad$ Early Pulmonary Lesions in Partially Immune Alcoholized Mice Following Inhalation of Virulent Pneumococci.

40. Blake, F. G., and Cecil, R. L., J. Exper. Med., 1920, xxxi, 403. Studies on Experimental Pneumonia. I. Production of Pneumococcus Lobar Pneumonia in Monkeys. J. Exper. Med., 1920, xxxi, 445. II. Pathology and Pathogenesis of Pneumococcus Lobar Pneumonia in Monkeys. J. Exper. Med., 1920, xxxi, 499. III. Spontaneous Pneumonia in Monkeys.

41. Cecil, R. L., and Blake, F. G., J. Exper: Med., 1920, xxxi, 519. IV. Results of Prophylactic Vaccination Against Pneumococcus Pneumonia in Monkeys.

42. Baldwin, E. R., and Gardner, L. U., Am. Rev. Tuberc., 1921, v, $429 . \quad$ Reinfection in Tuberculosis. Experimental Arrested Tuberculosis and Subsequent Infections.

43. Krause, A. K., and Peter, D., Am. Rev. Tuberc., 1920, iv, 551. Studies on Immunity to Tuberculosis. A Description of Graphic Records of the Local Allergic and Immune Reactions to Tuberculous Reinfection in Guinea Pigs.

44. Soper, W. B., Am. Rev. Tuberc., 1917, i, 385. Experimental Tuberculosis of the Liver.

45. Rich, A. R., and McCordock, H. A., Bull. Johns Hopkins Hosp., 1929, xliv, 273. An Enquiry Concerning the Role of Allergy, Immunity and Other Factors of Importance in the Pathogenesis of Human Tuberculosis.

46. Opie, E. L., J. Immunol., 1924, ix, 259. Pathogenesis of the Specific Inflammatory Reaction of Immunized Animals (Arthus Phenomenon) Relation of Local "Sensitization" to Immunity. 
47. Dochez, A. R., and Sherman, L., Proc. Soc. Exper. Biol., and Med., 1925, xxii, 282. Some Reactions in Sensitized Guinea Pigs to the Filtrate of Scarlatinal Streptococcus.

48. Dochez, A. R., and Stevens, F. A., J. Exper. Med., 1927, xlvi, 487. Studies on the Biology of Streptococcus. VII. Allergic Reactions with Strains from Erysipelas.

49. Cooke, J. V., Am. J. Dis. Child., 1927, xxxiv, 969. Scarlet Fever. I. The Relation Between Antitoxin in the Blood and Skin Sensitivity to Toxin in New-Born Infants and Their Mothers. Am. J. Dis. Child., 1928, xxxv, 762. II. The Development of Toxin Sensitivity of the Skin in Infants and its Relation to the Presence of Antitoxin in the Blood. Am. J. Dis. Child., 1928, xxxv, 991. VIII. The Anaphylactic Factor in Scarlet Fever.

50. Parker, J. T., J. Exper. Med., 1929, 1, 161. Experimental Pneumonia in Guinea Pigs. II. Effect of Anti-autolysate Sera on Pneumococcus Pneumonia in Guinea Pigs.

51. Smith, T., J. Med. Research, 1913, xxix, 291. Some Bacteriological and Environmental Factors in the Pneumonias of Lower Animals with Special Reference to the Guinea Pig.

52. Jordan, E. O., and Falk, I. S. Ed. The Newer Knowledge of Bacteriology and Immunology, Chicago, 1928. The Univ. of Chicago Press. Meyer, K. F., Communicable Diseases of Laboratory Animals. 